\title{
Monte-Carlo Modeling of Photoelectron Kinetics in Quantum-Dot Photodetectors
}

\author{
Vladimir Mitin, Andrei Sergeev, Li-Hsin Chien, and Nizami Vagidov \\ Electrical Engineering \\ University at Buffalo, The State University of New York \\ Buffalo, NY 14260 \\ e-mail:vmitin@buffalo.edu
}

\begin{abstract}
Using Monte-Carlo method, we simulate kinetics and transport of electrons in different types of InAs/GaAs quantum-dot infrared photodetectors. Our simulation program exploits $\Gamma$-L-X model of the conduction band of semiconductor and it includes three major types of electron scattering on: 1) acoustic phonons, 2) polar optical phonons, and 3) intervalley phonons. The results of simulation demonstrate that the combination of local potential barriers around quantum dots and quantum-dot structure with collective barriers can be used to achieve long carrier lifetimes, and therefore high photoconductive gain, responsivity, and detectivity.
\end{abstract}

Index Terms-Infrared photodetectors; quantum-dot structures; Monte-Carlo modeling

\section{INTRODUCTION}

Infrared photodetectors have attracted lots of attention recently because of their importance in environmental protection, medical and security applications. Particularly, quantumwell infrared photodetectors (QWIPs) have been extensively studied and they demonstrated successful imagery performance. However, QWIPs require low operating temperatures due to high thermionic emission rate. Moreover, they have inherent insensitivity to the normal incident radiation due to polarization selection rules. Recently, quantum-dot infrared photodetectors (QDIPs) (see Fig. 1) have been considered as a potential alternative to QWIPs because of their intrinsic sensitivity to normal incident radiation and longer carrier lifetimes, which provide higher photoconductive gain and higher operating temperatures. In the last few years, roomtemperature QDIPs have been demonstrated with detectivities $D^{*} \approx 10^{7}-10^{8} \mathrm{~cm} \mathrm{~Hz}^{1 / 2} / \mathrm{W}$ [1] - [3]. However, the QDIP's performance can be significantly improved by the proper choice of the device structure and by the selective doping.

\section{THEORY}

In order to increase the detectivity and responsivity of QDIPs, one should suppress the carrier capture by quantum dots (QDs). Therefore, one should separate the conducting electron states, which provide electron transport, from the localized states, which are excited by infrared (IR) radiation. The QDs in real space can be separated from the conducting states by the potential barriers, which prevent the carrier capture. In our papers [4] - [8] and [12], we have suggested that these separating potential barriers can be realized by: (a) homogeneous doping of the interdot space, which creates local

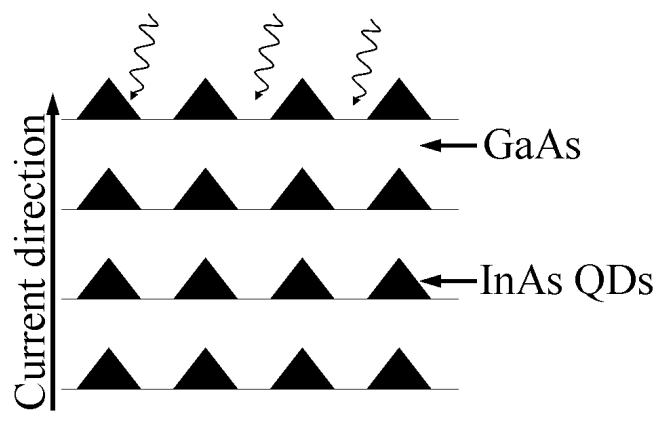

Fig. 1. Schematic cross-section of quantum-dot structure with vertical electron transport.

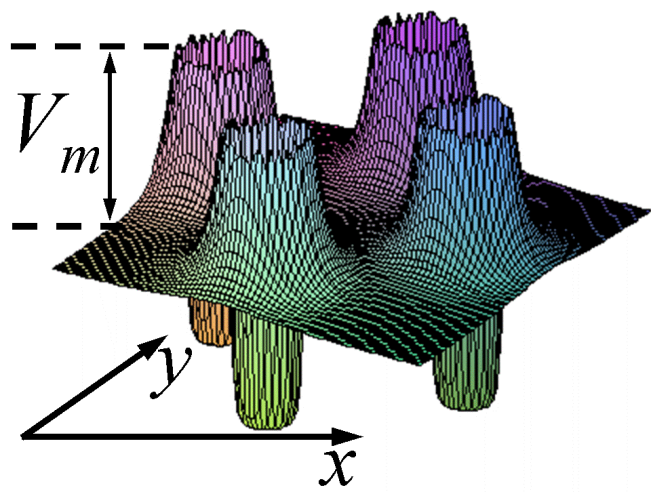

Fig. 2. Quantum-dot structure with the local potential barriers around quantum dots.

potential barriers around each quantum dot (Fig. 2) and (b) modulation doping of quantum-dot structure, which creates the collective potential barriers (Fig. 3). In the case of the collective barriers the electrons populating QDs are taken relatively far from the QDs. As it is shown in Fig. 3 the groups of QDs arranged in rows are separated from the undoped conducting channels, thus providing high electron mobility.

Nowadays, most of the QDIP structures reported utilize electron transport in the vertical direction through the layers of stacked QDs (see Fig. 1). However, because of the inherent inhomogeneity in size distribution of the self-assembled QDs, the transport through the layers of self-assembled QDs may 


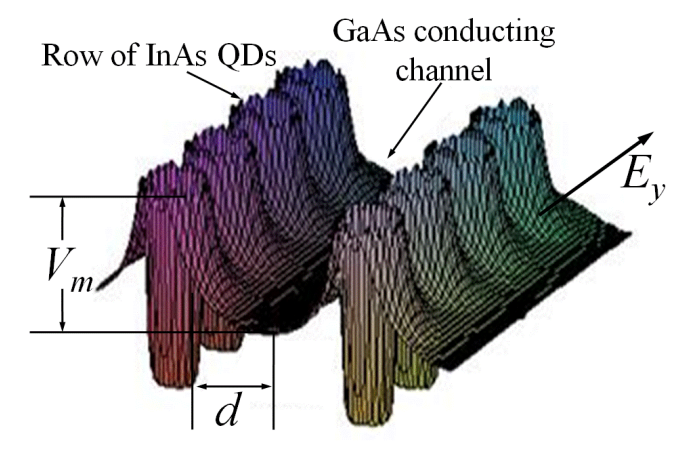

Fig. 3. Quantum-dot structure with collective barriers and conducting channels.

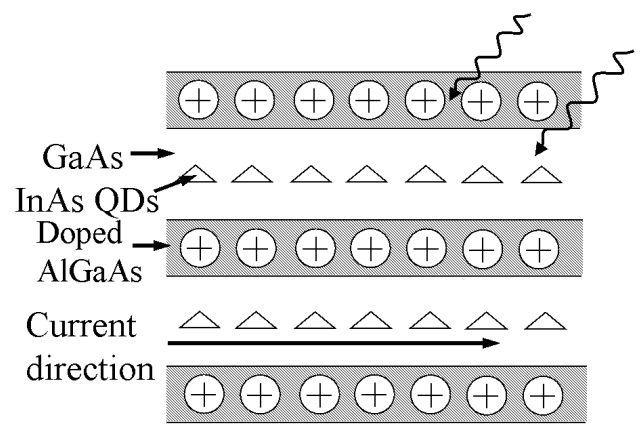

Fig. 4. Schematic cross-section of the modulation-doped quantum-dot structure with the lateral electron transport.

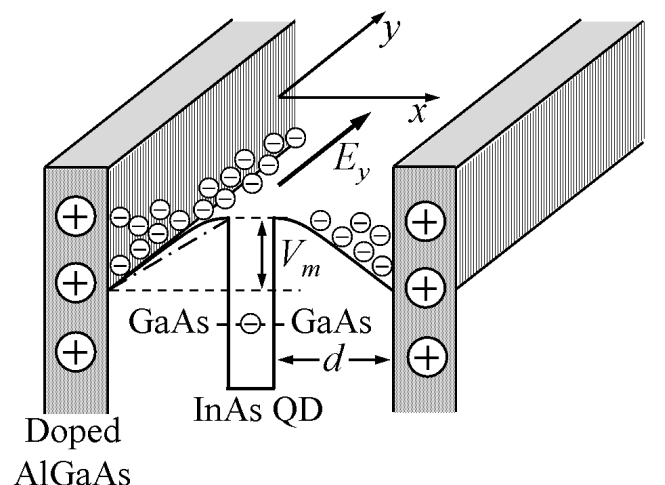

Fig. 5. Potential profile of the modulation-doped quantum-dot structure with the lateral electron transport.

be affected by the disorder. As a result, in this type of vertical transport structures, it might be difficult to achieve high electron mobility and high gain. Therefore, an alternative structure based on the lateral transport along the semiconductor heterointerfaces was suggested [9] - [11] (see, structure's cross-section and potential profile in Figs. 4 and 5). These modulation-doped quantum-dot infrared photodetectors are one of the possible realizations of structures with collective potential barriers. Due to large distances between QDs and heterointerfaces in such specific structures, the electron capture

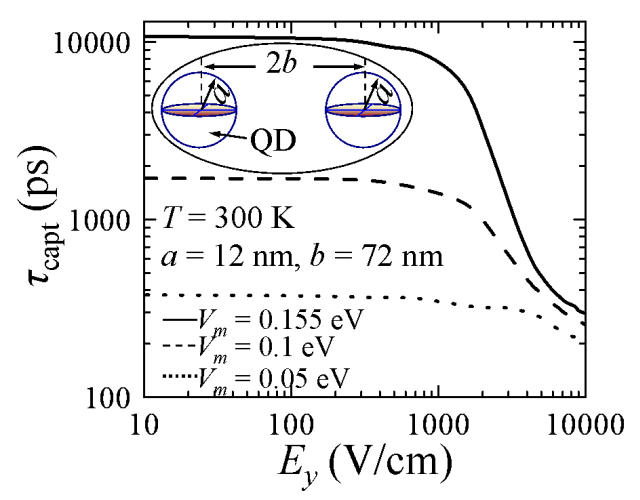

Fig. 6. Carrier capture time, $\tau_{\text {capt }}$, as a function of the longitudinal electric field, $E_{y}$, at different potential barrier heights, $V_{m}$, in the case of a quantumdot structure with local potential barriers around QDs. The inset shows the radius of a $\mathrm{QD}, a$, and interdot distance, $b$.

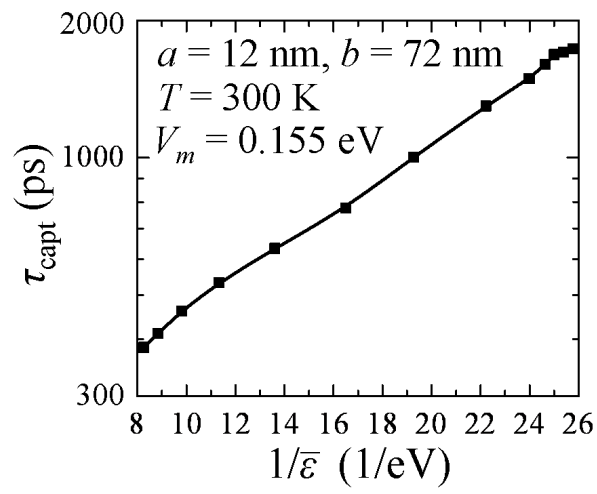

Fig. 7. Electron capture time, $\tau_{\text {capt }}$, as a function of the inverse electron average energy, $1 / \bar{\varepsilon}$, in the case of a quantum-dot structure with local potential barriers around QDs.

rates can be reduced and long electron lifetimes may be achieved, thus, providing high responsivity of the photodetector.

If there are no external fields, the capture rate in the presence of local potential barriers can be described by the following formula [12]:

$$
\frac{1}{\tau_{\text {capt }}}=\pi N_{d} a^{3} \frac{\tilde{v}}{\tilde{v}^{\prime}} \frac{1}{\tau_{\varepsilon}^{\prime}} \exp \left(-\frac{e V_{m}}{k T}\right),
$$

where $N_{d}$ is the concentration of quantum dots, $a$ is the radius of the dot, $\tilde{v}$ is the electron thermal velocity in the matrix surrounding quantum dots, $\tilde{v}^{\prime}$ is the electron thermal velocity in the dot, $\tau_{\varepsilon}^{\prime}$ is the relaxation time described by the inelastic intradot relaxation processes, and $V_{m}$ is the height of the potential barriers. As it follows from Eq. (1), the electron capture rate may be controlled by the variation of the height of the local potential barriers, $V_{m}$.

\section{Results of Simulation}

To study the effects of the electric field on the electron capture rate, we have developed simulation program based on the many-particle Monte-Carlo method. Our program includes three major types of scattering processes such as scattering 


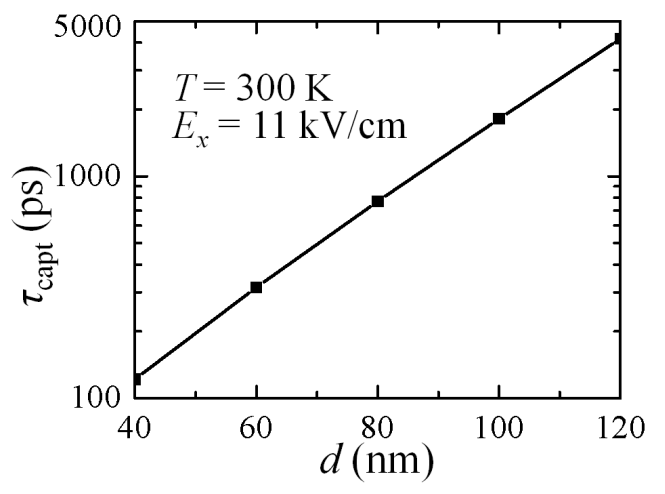

Fig. 8. Electron capture time, $\tau_{c a p t}$, as a function of GaAs thickness, $d$, in the case of a quantum-dot structure with collective potential barriers.

on acoustic, polar optical, and intervalley phonons. We use $\Gamma-L-X$-model of semiconductor for simulation of threedimensional electron transport in GaAs matrix with InAs dots.

In current paper we present the results of numerical simulations for three types of QDIP structures: (1) with local potential barriers around QDs (see, Fig. 2), (2) with collective barriers and conducting channels (see, Fig. 3), and (3) with combination of local and collective potential barriers. First, let us discuss the results of simulation for the first model with local potential barriers around QDs. Figure 6 presents the dependence of the electron capture time on the longitudinal electric field, $E_{y}$, for different heights of the potential barrier, $V_{m}$. This figure shows that the electron capture time, $\tau_{c a p t}$, is almost independent on $E_{y}$ up to the threshold field of the order of $1 \mathrm{kV} / \mathrm{cm}$. Once the electric field is higher than this threshold, the capture time drastically decreases with the increase of the field. Let us analyze the effect of the applied electric field on the electron transport from the point of view of electron heating. Figure 7 shows the exponential dependence of the capture time, $\tau_{\text {capt }}$, on the inverse average electron energy, $1 / \bar{\varepsilon}$. This result follows from Eq. (1), if the thermal energy $k T$ is replaced by a factor of $2 \bar{\varepsilon} / 3$. Thus, the effect of the electric field on the electron capture by QDs may be described in the framework of the model of electron heating.

Let us discuss the results obtained for the quantumdot structure with the collective barriers. Instead of a selfconsistent potential profile of the GaAs layer shown in Fig. 5 by a solid line, we have used slightly simplified potential profile (triangular approximation) shown in the same figure by the dash-dotted line. This triangular potential is equivalent to the existence of the constant perpendicular electric field, $E_{x}$. The value of $E_{x}$, utilized here, is equal to $11 \mathrm{kV} / \mathrm{cm}$. Figure 8 presents the dependence of $\tau_{\text {capt }}$ on the GaAs thickness, $d$, obtained for the quantum-dot structure with collective potential barriers in a triangular approximation. With the increase of $d$, electrons are more and more localized in the middle between quantum-dot rows, and $\tau_{\text {capt }}$ increases as $d$ increases. Figure 8 demonstrates the exponential dependence on $d$, which is in good agreement with the experimental results of [10].

Figure 9 shows the dependence of $\tau_{\text {capt }}$ on the applied

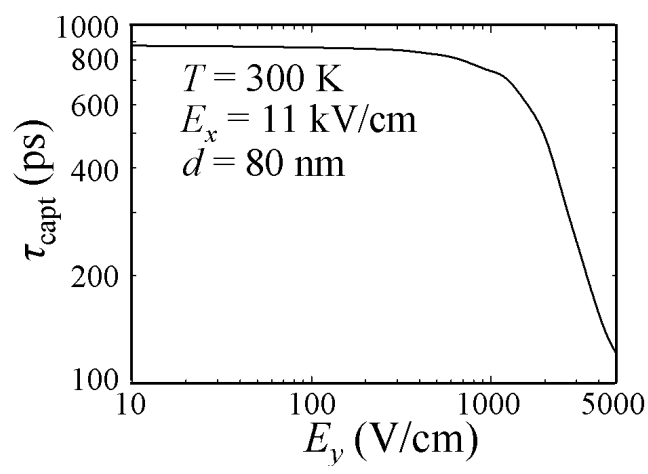

Fig. 9. Electron capture time, $\tau_{c a p t}$, as a function of longitudinal electric field, $E_{y}$, in the case of a quantum-dot structure with collective potential barriers.

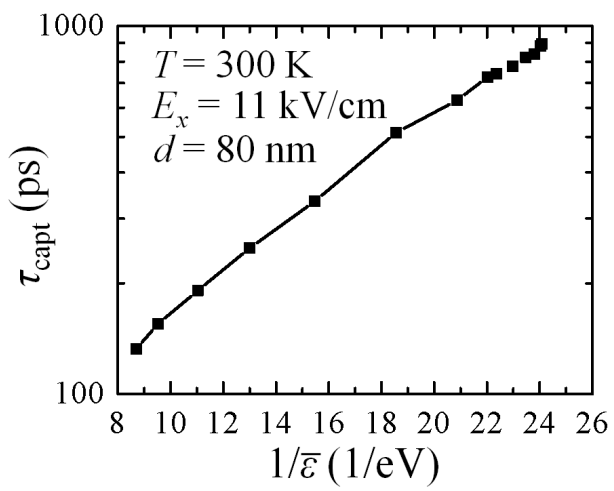

Fig. 10. Electron capture time, $\tau_{c a p t}$, as a function of inverse average energy $1 / \bar{\varepsilon}$, in the case of a quantum-dot structure with collective potential barriers.

longitudinal electric field, $E_{y}$, for the thickness of GaAs layer, $d$, equal to $80 \mathrm{~nm}$. This dependence has the same characteristic features as the dependences obtained for the model with the local potential barriers (compare with the dependence with $V_{m}$ $=0.1 \mathrm{eV}$ in Fig. 6).

Figure 10 shows the influence of electron heating on the capture time, $\tau_{\text {capt }}$, in the case of a quantum-dot structure with collective potential barriers. The result is almost the same as in Fig. 7, which was obtained for the model with local potential barriers. These results demonstrate that the model of electron heating can also be applied to the quantum-dot structures with collective potential barriers.

Let us analyze the characteristics of QDIP structure, which combines local and collective potential barriers. Figure 11 shows the dependence of the electron capture time, $\tau_{\text {capt }}$, on the applied longitudinal electric field, $E_{y}$, for such a structure. The electric field, $E_{x}$, which perpendicular to $E_{y}$, is equal to $11 \mathrm{kV} / \mathrm{cm}$. The dependence has the same characteristic features as for the considered above two structures (see Figs. 6 and 9). We see that for the model, which combines local and collective barriers, $\tau_{\text {capt }}$ is much higher. This might be used for the fabrication of optimal QDIP structures.

Figure 12 shows the exponential dependence of the capture time, $\tau_{\text {capt }}$, on the inverse average electron energy, $1 / \bar{\varepsilon}$, for 


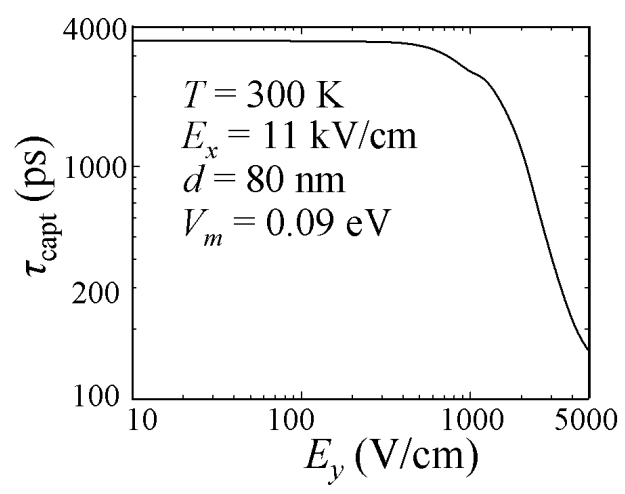

Fig. 11. Electron capture time, $\tau_{c a p t}$, as a function of longitudinal electric field, $E_{y}$, for the model, which combine local and collective potential barriers.

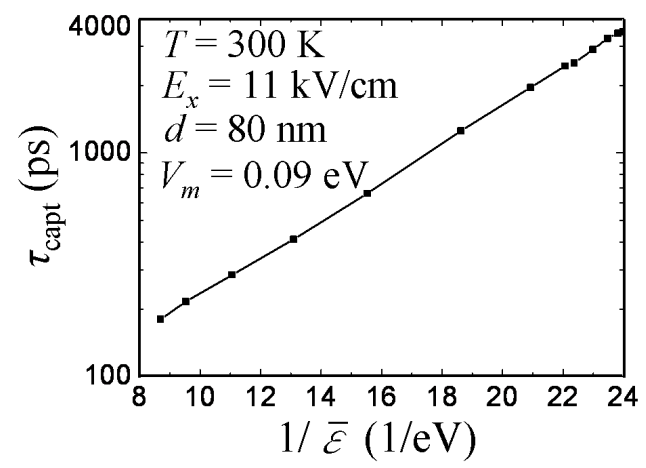

Fig. 12. Electron capture time, $\tau_{c a p t}$, as a function of inverse energy $1 / \bar{\varepsilon}$ for the model, which combine local and collective potential barriers.

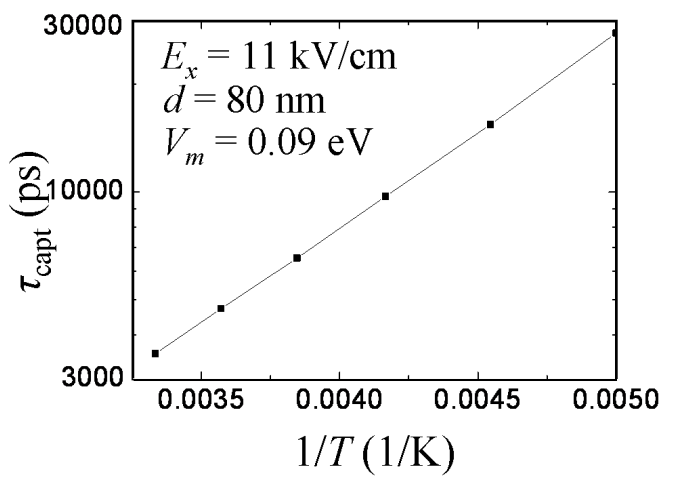

Fig. 13. The capture time, $\tau_{c a p t}$, as a function of the inverse temperature, $1 / T$, for the model, which combine local and collective potential barriers.

the model, which combines the local and collective potential barriers. This result has the same characteristic features as Figs. 7 and 10.

Currently, the operating temperatures of QDIPs is still a key issue. The obtained for different temperatures results (see Figure 13) have the exponential dependence of $\tau_{\text {capt }}$ on the inverse temperature, $1 / T$, which correlates with Eq. (1).

\section{CONCLUSION}

The local potential and collective barriers may be used to separate the localized intradot electron states from the conducting states in the matrix surrounding QDs, and, thus, to control the electron capture processes. The specific optimal structure can be realized by the combination of local and collective potential barriers in quantum-dot structures. With longer electron lifetimes the higher responsivity, detectivity, and photoconductive gain may be achieved. The manageable electron kinetics will allow one to employ quantum-dot sensors as adaptive detectors with controlled parameters.

\section{ACKNOWLEDGMENT}

This research was supported by AFOSR grant.

\section{REFERENCES}

[1] P. Bhattacharya, X. H. Su, S. Chakrabarti, G. Ariyawansa, and A. G. U. Perera, "Characteristics of a tunneling quantum-dot infrared photodetector operating at room temperature," Appl. Phys. Lett., vol. 86, pp. 191106-1-3, May 2005.

[2] S. Tsao, H. Lim, W. Zhang, and M. Razeghi, "High operating temperature 320256 middle-wavelength infrared focal plane array imaging based on an InAs/InGaAs/InAlAs/InP quantum dot infrared photodetector,' Appl. Phys. Lett., vol. 90, pp. 201109-1-3, May 2007.

[3] H. Lim, S. Tsao, W. Zhang, and M. Razeghi, "High-performance InAs quantum-dot infrared photodetectors grown on InP substrate operating at room temperature," Appl. Phys. Lett., vol. 90, pp. 131112-1-3, March 2007.

[4] V. V. Mitin, V.I. Pipa, A.V. Sergeev, M. Dutta, and M. Stroscio, "Highgain quantum-dot infrared photodetector,' Infrared Physics and Technol., vol. 42, pp. 467-472, June 2001.

[5] V. Ryzhii, I. Khmyrova, V. Mitin, M. Stroscio, and M. Willander, "On the detectivity of quantum-dot infrared photodetectors," Appl. Phys. Lett., vol. 78, pp. 3523-3525, May 2001.

[6] A. Sergeev, V. Mitin, and M. Stroscio, "Quantum-dot photodetector operating at room temperatures: diffusion-limited capture," Physica B vol. 316-317, 369-372, May 2002.

[7] V. Mitin, N. Vagidov, and A. Sergeev, "Infrared detector based on modulation doped quantum-dot structures," Physica Status Solidi C, vol. 3, pp. 4013-4016, November 2006.

[8] N. Vagidov, A. Sergeev, and V. Mitin, "Infrared quantum-dot detectors with diffusion-limited capture," International Journal of High Speed Electronics and Systems, vol. 17, pp. 585-591, September 2007.

[9] S.-W. Lee, K. Hirakawa, and Y. Shimada, "Bound to continuum intersubband photoconductivity of self-assembled InAs quantum dots in modulation-doped heterostructures," Appl. Phys. Lett., vol. 75, pp. 14281430, September 1999.

[10] K. Hirakawa, S.-W. Lee, Ph. Lelong, S. Fujimoto, K. Hirotanu, and H. Sakaki, "High-sensitivity modulation-doped quantum-dot infrared photodetectors," Microelectronic Engineering, vol. 63, pp. 185-192, August 2002.

[11] S.-W. Lee and K. Hirakawa, "Lateral conduction quantum-dot infrared photodetectors using photoionization of holes in InAs quantum dots,' Nanotechnology, vol. 17, pp. 3866-3868, August 2006.

[12] L. H. Chien, A. Sergeev, N. Vagidov, and V. Mitin, "Hot-electron transport in quantum-dot photodetectors," International Journal of High Speed Electronics and Systems, vol. 18, pp. 255-264, December 2008. 